\title{
COVID-19 vaccines: implementation, limitations and opportunities
}

\author{
Douglas D. Richman*
}

University of California San Diego, Distinguished Professor of Pathology and Medicine (Active Emeritus); Director, The HIV Institute; CoDirector, San Diego Center for AIDS Research; Florence Seeley Riford Emeritus Chair in AIDS Research, CA, USA.

\begin{abstract}
The speed of development and the magnitude of efficacy of recently developed vaccines directed against SARS-CoV-2 has been truly remarkable. This editorial will not summarize the highly publicized and reviewed information about the design and clinical trial results of these vaccines. Rather, I will speculate about several issues regarding $i$ ) considerations of the rollout and implementation of the multiple vaccines, ii) the use of the vaccines in ways different from those used in the registrational phase 3 studies, iii) the future both of SARS-CoV-2 in the human population and of "normal" human life returning after widespread vaccination, and $i v$ ) the implications of the success of these SARS-CoV-2 vaccines for vaccine development against other pathogens.
\end{abstract}

Keywords: COVID-19, SARS-CoV-2, vaccine

The speed of development and the degree of efficacy of vaccines directed against SARS-CoV-2 has exceeded expectations of the most optimistic of us. To proceed within a single calendar year from the availability of the viral sequence to the initiation of immunization of tens of millions of people in several countries is the scientific breakthrough of the decade (if not longer) (1). The Moderna and Pfizer/BioNTech mRNA-based vaccines are safe and $94-95 \%$ effective. The efficacy of various vaccines based on adenovirus vectors or viral protein preparations are rapidly becoming available. Up to 200 additional investigational vaccines are in pre-clinical or early clinical studies. Numerous reviews of the design, development and evaluation of all these products have been published and will not be recapitulated here (2). What I will address are speculations on several specific issues about the future that result from these remarkable accomplishments.

What is the utility and role of several different, potentially competing vaccines?

To date, all the available data indicate no safety concerns to preclude general use for any vaccines in phase 3 development. The efficacy of the two mRNA-based vaccines has exceeded any expectations $(3,4)$. The chimpanzee adenovirus-vectored Oxford/AstraZeneca vaccine (ChAdOx1) achieved 62\% efficacy in initial trials, thus not attaining the $94-95 \%$ level of protection of the mRNA vaccines (5); however, the Russian Sputnick $\mathrm{V}$ heterologous adenovirus 26 prime with adenovirus 5 boost resulted in $92 \%$ efficacy in the preliminary report of the phase 3 results (6). More results are anticipated. The preliminary phase 3 reports of the adenovirus 26 -based $\mathrm{J} \& \mathrm{~J} / \mathrm{Janssen}$ vaccine indicated a $72 \%$ efficacy against symptomatic disease in the United States and a $66 \%$ efficacy in all participating countries with an $85 \%$ efficacy against severe disease or death (7). It should be acknowledged that the various vaccine studies were performed at different times in different locations and thus cannot be directly compared. For example, a significant proportion of the study subjects in South Africa were infected with the new B.351 variant, which has been shown to be less susceptible to antibodies elicited with the original Wuhan strain of antigen, which is in the composition of all the vaccines currently being evaluated (see below). The results of the study in the United States of the Janssen vaccine evaluating the benefits of a booster injection are eagerly awaited. The Novavax nanoparticle, protein-based vaccine appears as least as immunogenic as the mRNA-based vaccines (8). Preliminary phase 3 results from the United Kingdom indicated $89 \%$ efficacy with over $50 \%$ of cases attributable to the more transmissible B.1.1.7 variant, and a phase $2 b$ trial in South Africa showed 60\% efficacy, in which approximately $90 \%$ of the endpoints occurred in subjects infected with the B.351 variant (9).

Consequently, multiple vaccines should be at our disposal to draw upon to quench the global pandemic. Even with the widespread application of the highly effective Pfizer and Moderna vaccines in the United States and several other countries, these vaccines will not be sufficient to immunize the majority of the 7 billion people on the planet. Even should other vaccines 
fall short of the $94-95 \%$ efficacy of these first two vaccines, multiple vaccines will be essential, and some may prove more appropriate for application among different populations living in different circumstances, especially low- and middle-income countries (LMIC). First, cost per dose of an adenovirus-vectored vaccine is approximately 2 US\$, while mRNA vaccines are approximately ten times more expensive. Second, the ultra-cold chain requirements for the mRNA-based vaccines are not feasible in most parts of less resourcerich countries, as well as in more rural and remote areas of the rest of the world. Third, billions of doses of vaccine are needed, and no vaccine manufacturer can produce sufficient vaccine alone. Fourth, the Janssen adenovirus 26-based vaccine has been originally tested with a single administration. For immunizing very large numbers of individuals, especially in LMIC, this would have very substantial benefits with regard to cost, health care resources and widespread rollout to benefit public health. This single-dose regimen would thus have numerous benefits, although a boosting injection does enhance immune responses and thus potential efficacy (10). Consequently, the implementation of global immunization will require multiple vaccines from different companies, and equally important, a rational strategy for selecting the most practical vaccine for each country, coordinated with allocation, distribution and administration processes to immunize as much of the world as expeditiously as possible. In the interests of public health, greater protection will result from immunizing many fold more members of the population with a vaccine conferring $70 \%$ efficacy than restricting use to a vaccine with $95 \%$ efficacy. These processes need to be conducted in parallel with information and educational efforts to overcome vaccine hesitancy and disinformation campaigns. Emerging data about safety, efficacy, production, cost, ease of administration, etc. will modulate decisions over time.

\section{What alternative regimens should be considered for the administration of these vaccines?}

With only millions of vaccine doses available in 2021 and billions of people in need of vaccination, alternative regimens for the use of the available vaccines have been proposed to stretch the supply to meet the demand and save lives. One is to administer half doses, for example of the Pfizer vaccine. This approach of halving the dose to double the available doses has proven very useful for addressing the recent yellow fever outbreaks (11); however, data to support the efficacy and durability of lower dose vaccine for COVID-19 have resulted in concern in the majority in the scientific community. Similarly, suggestions that different vaccines could be mixed for the prime and boost administrations have been proposed; however, once again there are simply no data regarding immunogenicity, safety or efficacy. It is conceivable there may be a benefit to such a strategy, especially since vector-based vaccines do elicit antivector immunity which dampens responses (12). This is in fact the rationale behind the Russian Sputnik $\mathrm{V}$ vaccine, which uses an adenovirus 26 prime and an adenovirus 5 boost, using the same SARS-CoV-2 spike construct (13). Nevertheless, without clinical trial data it is hard to justify employing this approach with different products; however, in cases of severe interruptions in vaccine supply, an argument can be made that individuals in need of a second dose should receive whatever vaccine is available.

Controversial with knowledgeable proponents on both sides is the proposal that, with inadequate supplies of vaccines early in the rollout, all available vaccine should be used to immunize as many people as possible with the first injection, while assuming that supplies for the second injection may be delayed by a month or two. Although the protocols for the registrational studies specified a 3-week interval between the two doses for the Pfizer vaccine and 4 weeks for the Moderna vaccine, from a public health point of view many more individuals would be protected early by not withholding half of the vaccine supply to insure availability of the second injection at the specified interval. The argument for withholding relies on the evidence from the 30,00044,000 person phase 3 trials that demonstrated efficacy according to protocols with just a few days of leeway with regard to the timing of the boosting dose and with no clinical trial data to show that a longer interval would be at least as effective or durable. The argument for immunizing as many as possible quickly with the possibility of a delayed second injection is based on several points: 1) The data show almost equivalent protection approximately 12 days after injection 1 for both Moderna and Pfizer, as for the period after dose 2. This means that with a disease incubation time of 5-7 days, protection is close to full after injection 1 for both vaccines within a week of exposure, consistent with the 5-7 day interval to detect neutralizing antibodies for most new antigens; 2) We do not know the durability of only 1 injection, but it is not showing any diminution at one month. Protecting twice as many people as fast as possible is better for public health than protecting half as many, even if there were a slight diminution of efficacy at least for several months (which I do not think is likely). The diminution will not be $50 \%$; 3 ) Delaying a boost beyond a month theoretically and in mice permits the peak and potentially interfering neutralizing antibody titer to decay while permitting $\mathrm{T}$ cell and perhaps B cell memory to mature (14). Boosts are less effective at $<3-4$ weeks following prime, but are as good as or better over the interval after one month for at least 6 months (and for measles for 4 years) (14). Recent data from the AstraZeneca ChAdOx1 trials suggest both better immune responses and increased protection the longer the interval between 
3 weeks and 3 months or more (15).Consequently, this writer supports the "first doses first" strategy, i.e., immunizing as many people as quickly as possible while awaiting additional supplies and information, acknowledging that this rationale is based on some extrapolations, rather than the data generated by clinical trials with a pre-specified injection interval.

\section{What will be the future of SARS-CoV-2 and of "normal" life returning after widespread vaccination?}

It is highly unlikely that SARS-CoV-2 will be extinguished like SARS-CoV-1. SARS-CoV-2 is clearly more transmissible than SARS-CoV-1, and is thus not amenable to the same transmission control measures. It can spread to the billions of remaining uninfected individuals on the planet, as well as the many millions who enter the population as a new birth cohort annually. The polio and measles eradication programs have struggled to eradicate these pathogens for decades because of the difficulties in reaching all corners of the globe, as well as the continual replenishment of new susceptibles by the annual birth cohort. Further complicating eradication are two factors, one shared with the polio and measles eradication challenges and one unique to SARS-CoV-2. As with all vaccination programs, vaccine hesitancy and misguided disinformation campaigns will diminish the penetration of protective vaccines in populations to sustain a large proportion of susceptibles. Unless overcome, this obstacle will confound the prospects of effective herd immunity. Unique to SARS-CoV-2 is the likelihood of the establishment of new animal reservoirs. The multiple introductions of SARS-CoV-2 into mink colonies with resulting transmission back into humans appears to threaten the industry of mink farming (16). Introductions into feline and canine species have been well documented, as well as the ability of the virus to infect numerous other species (17). Moreover, a chance transfer back into a bat population is not inconceivable. Consequently, it is almost certain that SARS-CoV-2 will not be extinguished and will likely remain a human pathogen, albeit controllable with effective vaccines.

One specter on the horizon, which may add to the threat of persistence of SARS-CoV-2, is the prospect of antigenic variants that escape immunity conferred by infection or vaccine. Several variants of concern have emerged since the Wuhan outbreak that appear to have occurred during human infection, rather than in a different species, like mink. The D614G and B.1.1.7 variants appear to be more fit by having been selected for increased transmissibility, but with no antigenic escape and possibly not significant virulence (18-20). Additionally concerning, are early data that indicate that the B.351 South African variant represents a neutralization escape variant to convalescent sera (21-23).

Speculation about the future applies to the lives of people as well as the virus. Will we be able to resume life as we knew it before COVID-19? To some extent, the pandemic has been sustained because many people never modulated their behavior during the threat of COVID-19. For many of us, who have been secluded at home and gone to work or shopping while exercising careful masking and other precautions, the opportunity to resume greater and more open contact with friends and family, to travel, and go to restaurants, theater and sporting events is greatly anticipated. Prolonging life and fully enjoying life should not be mutually exclusive. Following more widespread vaccination, many of us may gradually be able to resume most of these activities that we have avoided; however, there will likely be some permanent changes as the pandemic becomes controlled. As is the practice in many Asian societies, many individuals will increase their use of masks in public, not only for prevention of infection with SARS-CoV-2, but to reduce the risk of influenza and other respiratory virus infections, which appear to have diminished in incidence during the pandemic. Working remotely has had both benefits and frustrations. The benefits of reduced travel with cost savings for transportation and for meeting and office space will result in a greater proportion of work performed remotely than pre-pandemic. There may very well be substantial impacts on schooling, especially at higher levels and on business and scientific meetings. The loss of personal interactions at meetings (scientific, business, government and otherwise) has been a substantial loss, but remote meetings provide advantages of wider global participation, reduced costs, and diminished pollution and carbon footprint. More explicit predictions are well beyond the expertise of this writer.

\section{What scientific opportunities will be provided by the progress of COVID-19 vaccine accomplishments?}

The remarkable speed of COVID-19 vaccine development, clinical trials, emergency use authorization and rollout occurring in less than a year after the release of the virus sequence is remarkable and unprecedented. These accomplishments are a direct consequence of at least a decade of research in investigating new delivery platforms for HIV and other viruses. Of the first four vaccines developed in the United States and Western Europe, two were based on mRNA delivery and two on adenovirus vectors. What has been learned in the process of these successful vaccines, which extends over design, evaluation, production and delivery will certainly expand and accelerate vaccine development against the panoply of pathogens for which effective, or more effective, prevention is needed. A short list of pathogens that comprise this category includes HIV, influenza, respiratory syncytial virus (RSV), human 
metapneumovirus (hMPV), and other paramyxoviruses, dengue and other flaviviruses, rotavirus, Lassa virus, herpes simplex virus, cytomegalovirus, EpsteinBarr virus, hepatitis $\mathrm{C}$ virus, tuberculosis, syphilis, gonococcus, cholera, borreliosis, pertussis, rabies, and malaria. Some of these targets may not be amenable to the delivery of one or a few protein antigens and the appropriate antigen for many of these pathogens has not been defined. Nevertheless, the flexibility offered by these platforms, especially the mRNA-based platforms, and their implementation have now matured from unknowns to well-characterized, thus opening a generational opportunity for further applications to vaccines, the most effective and economical contribution of medical science to public health (24).

\section{Funding: None.}

Conflict of Interest: The author has no conflict of interest to disclose.

\section{References}

1. Cohen J. Shots of hope. Science. 2020; 370:1392-1394

2. Dai L, Gao GF. Viral targets for vaccines against COVID-19. Nat Rev Immunol. 2021; 21:73-82.

3. Polack FP, Thomas SJ, Kitchin N, et al. Safety and Efficacy of the BNT162b2 mRNA Covid-19 Vaccine. N Engl J Med. 2020; 383:2603-2615.

4. Baden LR, El Sahly HM, Essink B, et al. Efficacy and Safety of the mRNA-1273 SARS-CoV-2 Vaccine. N Engl J Med. 2021; 384:403-416.

5. Voysey M, Clemens SAC, Madhi SA, et al. Safety and efficacy of the ChAdOx1 nCoV-19 vaccine (AZD1222) against SARS-CoV-2: an interim analysis of four randomised controlled trials in Brazil, South Africa, and the UK. Lancet. 2021; 397:99-111.

6. Logunov DY, Dolzhikova IV, Shcheblyakov DV, et al. Safety and efficacy of an rAd26 and rAd5 vector-based heterologous prime-boost COVID-19 vaccine: an interim analysis of a randomised controlled phase 3 trial in Russia. Lancet. 2021. doi: https://doi.org/10.1016/S01406736(21)00234-8

7. Johnson \& Johnson Services, Inc. Johnson \& Johnson Announces Single-Shot Janssen COVID-19 Vaccine Candidate Met Primary Endpoints in Interim Analysis of its Phase 3 ENSEMBLE Trail. https://www.jnj.com/ johnson-johnson-announces-single-shot-janssen-covid19-vaccine-candidate-met-primary-endpoints-in-interimanalysis-of-its-phase-3-ensemble-trial (accessed January 28, 2021).

8. Keech C, Albert G, Cho I, et al. Phase 1-2 Trial of a SARS-CoV-2 Recombinant Spike Protein Nanoparticle Vaccine. N Engl J Med. 2020; 383:2320-2332.

9. Novavax, Inc. NOVAVAX. Novavax COVID-19 Vaccine Demonstrates $89.3 \%$ Efficacy in UK Phase 3 Trial. https:// ir.novavax.com/node/15506/pdf (accessed January 28, 2021).

10. Folegatti PM, Ewer KJ, Aley PK, et al. Safety and immunogenicity of the ChAdOx $1 \mathrm{nCoV}-19$ vaccine against SARS-CoV-2: a preliminary report of a phase $1 / 2$, single-blind, randomised controlled trial. Lancet. 2020; 396:467-478.

11. Juan-Giner A, Kimathi D, Grantz KH, et al. Immunogenicity and safety of fractional doses of yellow fever vaccines: a randomised, double-blind, non-inferiority trial. Lancet. 2021; 397:119-127.

12. Harro CD, Robertson MN, Lally MA, et al. Safety and immunogenicity of adenovirus-vectored near-consensus HIV type 1 clade B gag vaccines in healthy adults. AIDS Res Hum Retroviruses. 2009; 25:103-114.

13. Logunov DY, Dolzhikova IV, Zubkova OV, et al. Safety and immunogenicity of an rAd26 and rAd5 vector-based heterologous prime-boost COVID-19 vaccine in two formulations: two open, non-randomised phase 1/2 studies from Russia. Lancet. 2020; 396:887-897.

14. Sallusto F, Lanzavecchia A, Araki K, Ahmed R. From vaccines to memory and back. Immunity. 2010; 33:451463.

15. Voysey M, Costa Clemens SA, Madhi SA, et al. Single Dose Administration, And The Influence Of The Timing Of The Booster Dose On Immunogenicity and Efficacy Of ChAdOx1 nCoV-19 (AZD1222) Vaccine. 2021. Preprints with The Lancet. https://ssrn.com/abstract $=3777268$ (accessed February 3, 2021)

16. Oude Munnink BB, Sikkema RS, Nieuwenhuijse DF, et al. Transmission of SARS-CoV-2 on mink farms between humans and mink and back to humans. Science. 2021; 371:172-177.

17. Centers for Disease Control and Prevention. COVID-19 and Animals. https://www.cdc.gov/coronavirus/2019ncov/daily-life-coping/animals.html (accessed January 16, 2021).

18. Korber B, Fischer WM, Gnanakaran S, et al. Tracking Changes in SARS-CoV-2 Spike: Evidence that D614G Increases Infectivity of the COVID-19 Virus. Cell. 2020; 182:812-827.e19.

19. Volz E, Hill V, McCrone JT, et al. Evaluating the Effects of SARS-CoV-2 Spike Mutation D614G on Transmissibility and Pathogenicity. Cell. 2021; 184:64-75. e11.

20. Chand M, Hopkins S, Dabrera G, Achison C, Barclay W, Ferguson N, Volz E, Loman N, Rambaut A, Barrett J. Investigation of novel SARS-COV-2 variant: Variant of Concern 202012/01. https://assets.publishing.service.gov. uk/government/uploads/system/uploads/attachment_data/ file/947048/Technical_Briefing_VOC_SH_NJL2_SH2.pdf (accessed January 16, 2021).

21. Wibmer CK, Ayres F, Hermanus T, et al. SARS-CoV-2 501 Y.V2 escapes neutralization by South African COVID-19 donor plasma. https://www.biorxiv.org/co ntent/10.1101/2021.01.18.427166v1. doi: https://doi. org/10.1101/2021.01.18.427166

22. Andreano E, Piccini G, Licastro D, et al. SARS-CoV-2 escape in vitro from a highly neutralizing COVID-19 convalescent plasma. https://www.biorxiv.org/cont ent/10.1101/2020.12.28.424451v1. doi: https://doi. org/10.1101/2020.12.28.424451

23. Tegally H, Wilkinson E, Giovanetti M, et al. Emergence and rapid spread of a new severe acute respiratory syndrome-related coronavirus 2 (SARS-CoV-2) lineage with multiple spike mutations in South Africa. https:// www.medrxiv.org/content/10.1101/2020.12.21.2024864 0v1. doi: https://doi.org/10.1101/2020.12.21.20248640

24. Rappuoli R, De Gregorio E, Del Giudice G, Phogat S, Pecetta S, Pizza M, Hanon E. Vaccinology in the 
post-COVID-19 era. Proc Natl Acad Sci U S A. 2021; 118:e2020368118.

Received January 31, 2021; Accepted February 4, 2021.

Released online in J-STAGE as advance publication February $11,2021$.
*Address correspondence to:

Douglas D. Richman, University of California San Diego, Distinguished Professor of Pathology and Medicine (Active Emeritus); Director, The HIV Institute; Co-Director, San Diego Center for AIDS Research; Florence Seeley Riford Emeritus Chair in AIDS Research. Room 329, Stein Clinical Sciences Building, 9500 Gilman Drive, La Jolla, CA 92093-0679, USA. E-mail: drichman@health.ucsd.edu 